\title{
Estimates of the Fundamental Solution for Higher Order Schrödinger Type Operators and Their Applications
}

\author{
Satoko Sugano \\ Kobe City College of Technology, 8-3 Gakuen-higashimachi, Nishi-ku, Kobe 651-2194, Japan \\ Correspondence should be addressed to Satoko Sugano; sugano@kobe-kosen.ac.jp
}

Received 3 October 2013; Accepted 21 November 2013

Academic Editor: Hendra Gunawan

Copyright (C) 2013 Satoko Sugano. This is an open access article distributed under the Creative Commons Attribution License, which permits unrestricted use, distribution, and reproduction in any medium, provided the original work is properly cited.

We consider higher order Schrödinger type operators with nonnegative potentials. We assume that the potential belongs to the reverse Hölder class which includes nonnegative polynomials. We establish estimates of the fundamental solution and show $L^{p}$ boundedness of some Schrödinger type operators.

\section{Introduction}

Let $V(x)$ be a nonnegative potential and consider the Schrödinger type operators $H_{k}=(-\Delta)^{k}+V^{k}$ on $\mathbf{R}^{n}$, where $k$ is a positive integer and $n \geq 2 k+1$. When $V$ is a nonnegative polynomial, Zhong proved estimates of the fundamental solution for $H_{1}$ and $H_{2}$ and showed some estimates for $H_{1}$ and $H_{2}$ (see [1]). More precisely, he showed the $L^{p}$ boundedness of the operators $V H_{1}^{-1}, V^{1 / 2} \nabla H_{1}^{-1}$, and $V^{2-j / 2} \nabla^{j} H_{2}^{-1}$, where $j=0,1,2,3,4$. He also proved that the operators $\nabla^{2} H_{1}^{-1}$ and $\nabla^{4} \mathrm{H}_{2}^{-1}$ are Calderón-Zygmund operators. Recently, in [2], the authors showed the $L^{p}$ boundedness of the operators $V^{k} H_{k}^{-1}$, $\nabla^{2 k} H_{k}^{-1}$, and $\nabla^{k} H_{k}^{-1 / 2}$ for nonnegative polynomial potential $V$.

For the potential $V$ which belongs to the reverse Hölder class, which includes nonnegative polynomials, Shen generalized Zhong's results on $H_{1}$ (see [3]). Actually, he established estimates of the fundamental solution for $H_{1}$ and showed the $L^{p}$ estimates for the operators $V H_{1}^{-1}, V^{1 / 2} \nabla H_{1}^{-1}, \nabla^{2} H_{1}^{-1}$, and so on. For the operator $H_{1}$ with reverse Hölder class potentials, further results have been investigated by many researchers. See [4-7], for example. For the operator $H_{2}$ with reverse Hölder class potentials, in [8], the author established estimates of the fundamental solution for $\mathrm{H}_{2}$ and showed the $L^{p}$ boundedness of the operators $V^{2-j / 2} \nabla^{j} H_{2}^{-1}$, where $j=$
$0,1,2,3,4$. In [8], the author also showed that the operator $\nabla^{4} \mathrm{H}_{2}^{-1}$ is a Calderón-Zygmund operator. Recently, in [9], the authors showed $L^{p}$ boundedness of the operator $\nabla^{2} H_{2}^{-1 / 2}$ and that the operator $\nabla^{2} H_{2}^{-1 / 2}$ is a Calderón-Zygmund operator.

In this paper, we study $H_{2^{m}}=(-\Delta)^{2^{m}}+V^{2^{m}}$ with reverse Hölder class potential $V$ on $\mathbf{R}^{n}$, where $m$ is a nonnegative integer and $n \geq 2^{m+1}+1$. We establish estimates of the fundamental solution for $H_{2^{m}}$ and show the $L^{p}$ boundedness of the operators $V^{2^{m}-j / 2} \nabla^{j} H_{2^{m}}^{-1}$, where $j$ is an integer satisfying $0 \leq j \leq 2^{m+1}$.

As mentioned above, in [2], the authors proved some results on $H_{k}=(-\Delta)^{k}+V^{k}$, where $V$ is a nonnegative polynomial and $k$ is an integer, $k \geq 3$. They proved their results by making use of [2, Lemma 3.3 and Corollary 3.1] which have been proved only for nonnegative polynomial $V$. In this paper, our strategy is different from the one in [2], since the question whether the above two results can be proved for reverse Hölder class potentials $V$ is yet to be settled. The purpose of this paper is to show some results on $H_{k}=(-\Delta)^{k}+V^{k}$ with potential $V$ which belongs to the reverse Hölder class, which includes nonnegative polynomials. However, our results are only for $k=2^{m}$, where $m$ is an integer, $m \geq 2$.

We recall the definitions of the reverse Hölder class (e.g., [3]). We denote by $B_{r}(x)$ the ball centered at $x$ with radius $r$. 
Definition 1 (reverse Hölder class). Let $V \geq 0$.

(1) For $1<p<\infty$ one says that $V \in(R H)_{p}$ if $V \in$ $L_{\text {loc }}^{p}\left(\mathbf{R}^{n}\right)$ and there exists a positive constant $C$ such that

$$
\left(\frac{1}{\left|B_{r}(x)\right|} \int_{B_{r}(x)} V(y)^{p} d y\right)^{1 / p} \leq \frac{C}{\left|B_{r}(x)\right|} \int_{B_{r}(x)} V(y) d y
$$

holds for every $x \in \mathbf{R}^{n}$ and $0<r<\infty$.

(2) One says that $V \in(R H)_{\infty}$ if $V \in L_{\mathrm{loc}}^{\infty}\left(\mathbf{R}^{n}\right)$ and there exists a positive constant $C$ such that

$$
\|V\|_{L^{\infty}\left(B_{r}(x)\right)} \leq \frac{C}{\left|B_{r}(x)\right|} \int_{B_{r}(x)} V(y) d y
$$

holds for every $x \in \mathbf{R}^{n}$ and $0<r<\infty$.

Remark 2. (1) If $P(x)$ is a polynomial and $\alpha>0$, then $V(x)=$ $|P(x)|^{\alpha}$ belongs to $(R H)_{\infty}$ (see [10, page 146$\left.]\right)$.

(2) For $1<p<\infty$, it is easy to see $(R H)_{\infty} \subset(R H)_{p}$.

Definition 3 (see [3, Definition 1.3]). (1) Let $V \in(R H)_{n / 2}$ and $V \not \equiv 0$. Then it is well known that there exists a positive $\epsilon$ such that $V \in(R H)_{n / 2+\epsilon}$ (see [11, Lemma 2]). Then the function $\rho(x, V)$ is well defined by

$$
\frac{1}{\rho(x, V)}=\sup \left\{r>0: \frac{r^{2}}{\left|B_{r}(x)\right|} \int_{B_{r}(x)} V(y) d y \leq 1\right\}
$$

and satisfies $0<\rho(x, V)<\infty$ for every $x \in \mathbf{R}^{n}$.

One recalls that

$$
\frac{r^{2}}{\left|B_{r}(x)\right|} \int_{B_{r}(x)} V(y) d y=1 \quad \text { for } r=\frac{1}{\rho(x, V)}
$$

(see [3, page 519]).

Remark 4. (1) If $V \in(R H)_{p}, p \geq n / 2$, then there exists a positive constant $C$ such that

$$
\left(\frac{1}{\left|B_{r}(x)\right|} \int_{B_{r}(x)} V(y)^{p} d y\right)^{1 / p} \leq C \rho(x, V)^{2}
$$

(cf. [3, Lemma 1.8] and [5, Lemma 2.2 (a)]).

(2) If $V \in(R H)_{\infty}$ then there exists a positive constant $C$ such that $V(x) \leq C \rho(x, V)^{2}$ (see [3, Remark 2.9]).

Let $\alpha=\left(\alpha_{1}, \ldots, \alpha_{n}\right)$ denote the multi-index with $\alpha_{i} \in \mathbf{N}$, $i \in \mathbf{N}$, and $1 \leq i \leq n$. Define $x^{\alpha}=x_{1}^{\alpha_{1}} \cdots x_{n}^{\alpha_{n}}$ and $\nabla^{j}=$ $\partial^{\alpha}=\partial^{|\alpha|} /\left(\partial x_{1}^{\alpha_{1}} \cdots \partial x_{n}^{\alpha_{n}}\right)$ for $j=|\alpha|=\alpha_{1}+\cdots+\alpha_{n}$. For any positive integer $j$ and a function $u \in C^{j}\left(\mathbf{R}^{n}\right)$, denote that $\nabla^{j} u(x)=\left(\partial^{\alpha} u(x):|\alpha|=j\right)$ and $\left|\nabla^{j} u(x)\right|^{2}=\sum_{|\alpha|=j}\left|\partial^{\alpha} u(x)\right|^{2}$. We denote by $\Gamma_{H_{k}}(x, y)$ the fundamental solution for $H_{k}$. The operator $H_{k}^{-1}$ is the integral operator with $\Gamma_{H_{k}}(x, y)$ as its kernel.

Now we state our theorems.

Theorem 5. Let $j, m$, and $n$ be integers, $m \geq 0, n \geq 2^{m+1}+1$, and $0 \leq j \leq 2^{m+1}-1$. Suppose that $V \in(R H)_{q_{0}}$ for some $q_{0} \geq 2^{m} n /\left(2^{m+1}-j\right)$. Then there exist positive constants $C_{j}$ such that

$$
\left\|V^{2^{m}-j / 2} \nabla^{j} H_{2^{m}}^{-1} f\right\|_{L^{p}\left(\mathbf{R}^{n}\right)} \leq C_{j}\|f\|_{L^{p}\left(\mathbf{R}^{n}\right)}
$$

where $1 \leq p \leq 2 q_{0} /\left(2^{m+1}-j\right)$.

In Theorem 5 , the case $m=0$ was shown in [3, Theorems 2.7 and 4.13].

Remark 6. In Theorem 5, for the case $m=j=0$, inequality (6) was shown in [3, Remark 2.9] under the assumption $V \in$ $(R H)_{\infty}$. If we take the limit $q_{0} \rightarrow \infty$, then class $(R H)_{q_{0}}$ becomes $(R H)_{\infty}$ and $V \in(R H)_{\infty}$ implies " $V \in(R H)_{n / 2}$ and $V(x) \leq C \rho(x, V)^{2}$ ". See [8, Remark 3] and also [8, Theorem $1]$.

Remark 7. We assume " $V \in(R H)_{n / 2}$ and $V(x) \leq C \rho(x, V)^{2}$." Then inequality (6) is true for $1 \leq p \leq \infty$. See Section 7 for the details. We also remark that the inequality (6) is true for $j=2^{m+1}$ and $1<p<\infty$ under the assumption " $V \epsilon$ $(R H)_{n / 2}$ and $V(x) \leq C \rho(x, V)^{2}$ ". See [3, Remark 2.9] and also $[8$, Theorem $1(2)]$.

Theorem 8. Let $j, m$, and $n$ be integers, $m \geq 0, n \geq 2^{m+1}+1$, and $1 \leq j \leq 2^{m+1}-1$. Suppose that $V \in(R H)_{q_{0}}$ for some $q_{0}$, $n / 2 \leq q_{0}<2^{m} n /\left(2^{m+1}-j\right)$. Then there exist positive constants $C_{j}$ such that (6) holds, where $1 \leq p \leq p_{0}$ and $1 / p_{0}=\left(2^{m+2}-\right.$ $j) /\left(2 q_{0}\right)-\left(2^{m+1}-j\right) / n$.

To prove Theorems 5 and 8 we need the estimates of the fundamental solution (Theorems 9 and 10). The following Theorem 9 generalizes the results in [2, Theorem 3.1] to the operator $H_{2}$ with potential $V$ which belongs to the reverse Hölder class.

Theorem 9. Let $m$ and $n$ be integers, $m \geq 0$, and $n \geq 2^{m+1}+1$. Suppose that $V \in(R H)_{n / 2}$. Then for any positive integer $N$ there exists a positive constant $C_{N}$ such that

$$
(0 \leq) \Gamma_{H_{2^{m}}}(x, y) \leq \frac{C_{N}}{\{1+\rho(x, V)|x-y|\}^{N}} \cdot \frac{1}{|x-y|^{n-2^{m+1}}}
$$

In Theorem 9, the cases $m=0$ and $m=1$ were shown in [3, Theorem 2.7] and [8, Theorem 2], respectively. We prove Theorem 9 by induction; that is, we assume that Theorem 9 is true for $m=l$ and show the case $m=l+1$. We also prove the following theorem which states derivative estimates of the fundamental solution.

Theorem 10. Let $j, m$, and $n$ be integers, $m \geq 0, n \geq 2^{m+1}+1$, and $1 \leq j \leq 2^{m+1}-1$. Suppose that $V \in(R H)_{2^{m} n /\left(2^{m+1}-j\right)}$. Then for any positive integer $N$ there exists a positive constant $C_{N}$ such that

$$
\left|\nabla_{x}^{j} \Gamma_{H_{2^{m}}}(x, y)\right| \leq \frac{C_{N}}{\{1+\rho(x, V)|x-y|\}^{N}} \cdot \frac{1}{|x-y|^{n-2^{m+1}+j}} .
$$


In Theorem 10, the cases $m=0$ and $m=1$ were shown in [3, page 537] and [8, Theorem 6], respectively.

The plan of this paper is as follows. In Section 2, we describe some lemmas needed later. In Section 3, we assume that Theorem 9 is true for $m=l$ and show some estimates for $H_{2^{l}}$ which are needed to prove the case $m=l+1$ in Theorem 9. In Section 4, we prove the case $m=l+1$ in Theorem 9. Section 5 is devoted to proof of Theorem 10. In Section 6, we prove Theorems 5 and 8. Finally, in Section 7, we state some remarks. Throughout this paper the letter $C$ stands for a constant not necessarily the same at each occurrence.

\section{Preliminaries}

In this section, we describe some lemmas needed later. First, we remark that $V \in(R H)_{q}, q>1$, implies that $V(x) d x$ is a doubling measure; that is, there exists a positive constant $C_{0}$ such that

$$
\int_{B_{2 r}(x)} V(y) d y \leq C_{0} \int_{B_{r}(x)} V(y) d y
$$

(see [3, page 518] and also [5, Remark $1.2(1)]$ ).

Lemma 11 (see [3, Lemma 1.2]). Assume that $V \in(R H)_{q}$ for some $q>n / 2$. Then there exists a positive constant $C$ such that, for $0<r<R<\infty$,

$$
\frac{r^{2}}{\left|B_{r}(x)\right|} \int_{B_{r}(x)} V(y) d y \leq C\left(\frac{R}{r}\right)^{n / q-2} \frac{R^{2}}{\left|B_{R}(x)\right|} \int_{B_{R}(x)} V(y) d y .
$$

Lemma 12 (see [3, Lemma 1.4 (b), (c)]). (1) Suppose that $V \in$ $(R H)_{n / 2}$. Then there exist positive constants $C$ and $k_{0}$ such that, for $x, y \in \mathbf{R}^{n}$,

$$
\rho(y, V) \leq C\{1+\rho(x, V)|x-y|\}^{k_{0}} \rho(x, V) .
$$

(2) Suppose $V \in(R H)_{n / 2}$. Then there exist positive constants $C^{\prime}$ and $k_{0}$ such that, for $x, y \in \mathbf{R}^{n}$,

$$
\rho(y, V) \geq \frac{C^{\prime} \rho(x, V)}{\{1+\rho(x, V)|x-y|\}^{k_{0} /\left(k_{0}+1\right)}} .
$$

Lemma 13 (Caccioppoli type inequality). Let $a, j$, and $k$ be integers, $k \geq 2,1 \leq a \leq k / 2$, and $1 \leq j \leq 2 a$. Assume that $(-\Delta)^{k} u+V^{k} u=0$ in $B_{R}\left(x_{0}\right)$ for some $x_{0} \in \mathbf{R}^{n}$. Then there exists a positive constant $C$ such that

$$
\begin{aligned}
\int_{B_{R / 2}\left(x_{0}\right)}\left|\Delta^{k-a} u(x)\right|^{2} d x & \\
& +\int_{B_{R / 2}\left(x_{0}\right)} V(x)^{k}|u(x)|\left|\Delta^{k-2 a} u(x)\right| d x \\
\leq & C \sum_{j=1}^{2 a} \frac{1}{R^{2 j}} \int_{B_{R}\left(x_{0}\right)}\left|\nabla^{2 a-j}\left(\Delta^{k-2 a} u\right)(x)\right|^{2} d x .
\end{aligned}
$$

For readers' convenience, we give the proof of Lemma 13 at the end of this section. If $k$ is an even number, then letting $a=k / 2$ in Lemma 13 , we have the following.
Corollary 14 (see [2, Lemma 3.5]). Let $j$ be an integer, $k$ an even number, $k \geq 2$, and $0 \leq j \leq k-1$. Assume that $(-\Delta)^{k} u+V^{k} u=0$ in $B_{R}\left(x_{0}\right)$ for some $x_{0} \in \mathbf{R}^{n}$. Then there exists a positive constant $C$ such that

$$
\begin{gathered}
\int_{B_{R / 2}\left(x_{0}\right)}\left|\Delta^{k / 2} u(x)\right|^{2} d x+\int_{B_{R / 2}\left(x_{0}\right)} V(x)^{k}|u(x)|^{2} d x \\
\leq C \sum_{j=0}^{k-1} \frac{1}{R^{2(k-j)}} \int_{B_{R}\left(x_{0}\right)}\left|\nabla^{j} u(x)\right|^{2} d x .
\end{gathered}
$$

Lemma 15 (Caccioppoli type inequality). Let $a, j$, and $k$ be integers, $k \geq 1,1 \leq a \leq(k+1) / 2$, and $1 \leq j \leq 2 a-1$. Assume that $(-\Delta)^{k} u+V^{k} u=0$ in $B_{R}\left(x_{0}\right)$ for some $x_{0} \in \mathbf{R}^{n}$. Then there exists a positive constant $C$ such that

$$
\begin{aligned}
& \int_{B_{R / 2}\left(x_{0}\right)}\left|\nabla\left(\Delta^{k-a} u\right)(x)\right|^{2} d x \\
& +\int_{B_{R / 2}\left(x_{0}\right)} V(x)^{k}|u(x)|\left|\Delta^{k-2 a+1} u(x)\right| d x \\
& \leq C \sum_{j=1}^{2 a-1} \frac{1}{R^{2 j}} \int_{B_{R}\left(x_{0}\right)}\left|\nabla^{2 a-j-1}\left(\Delta^{k-2 a+1} u\right)(x)\right|^{2} d x .
\end{aligned}
$$

If $k$ is an odd number, then letting $a=(k+1) / 2$ in Lemma 15 , we have the following.

Corollary 16 (see [2, Lemma 3.5]). Let $j$ be an integer, $k$ an odd number, $k \geq 1$, and $0 \leq j \leq k-1$. Assume that $(-\Delta)^{k} u+V^{k} u=0$ in $B_{R}\left(x_{0}\right)$ for some $x_{0} \in \mathbf{R}^{n}$. Then there exists a positive constant $C$ such that

$$
\begin{gathered}
\int_{B_{R / 2}\left(x_{0}\right)}\left|\nabla\left(\Delta^{(k-1) / 2} u\right)(x)\right|^{2} d x+\int_{B_{R / 2}\left(x_{0}\right)} V(x)^{k}|u(x)|^{2} d x \\
\leq C \sum_{j=0}^{k-1} \frac{1}{R^{2(k-j)}} \int_{B_{R}\left(x_{0}\right)}\left|\nabla^{j} u(x)\right|^{2} d x .
\end{gathered}
$$

Lemma 17. Let $j, k$, and $n$ be integers, $k \geq 1, n \geq 2 k+1$, and $0 \leq j \leq k-1$. Assume that $(-\Delta)^{k} u+V^{k} u=0, u \geq 0$, in $B_{R}\left(x_{0}\right)$ for some $x_{0} \in \mathbf{R}^{n}$. Then there exists a positive constant $C$ such that

$$
\begin{aligned}
& \sup _{y \in B_{R / 2}\left(x_{0}\right)}|u(y)| \\
& \quad \leq C \sum_{j=0}^{k-1} R^{j}\left(\frac{1}{\left|B_{R}\left(x_{0}\right)\right|} \int_{B_{R}\left(x_{0}\right)}\left|\nabla^{j} u(x)\right|^{2} d x\right)^{1 / 2} .
\end{aligned}
$$

In Lemma 17, the cases $k=1$ and $k=2$ have been proved (see [3, page 523] and [1, Corollary 5.6]).

Proof of Lemma 17. We proceed following the proof of [1, Corollary 5.6]. We prove Lemma 17 for $u$ which satisfies $(-\Delta)^{k} u+V^{k} u=0, u \geq 0$, in $B_{R}\left(x_{0}\right)$. Let $a$ and $b$ be nonnegative integers and choose $\eta \in C_{0}^{\infty}\left(B_{R / 4}\left(x_{0}\right)\right)$ such that $\eta \equiv 1$ 
on $B_{R / 8}\left(x_{0}\right)$ and $\left|\nabla^{b}\left(\Delta^{a} \eta\right)\right| \leq C / R^{2 a+b}$. Let $\Gamma_{H_{k-1}, 0}(x, y)=$ $C|x-y|^{2(k-1)-n}$ be the fundamental solution for $(-\Delta)^{k-1}$. Note that

$$
\begin{aligned}
\Delta^{k-1} & (u(y) \eta(y)) \\
& =\sum_{a, b}\left(\begin{array}{c}
2(k-1) \\
2 a+b
\end{array}\right) \nabla^{b}\left(\Delta^{k-1-a-b} u\right)(y) \cdot \nabla^{b}\left(\Delta^{a} \eta\right)(y),
\end{aligned}
$$

where

$$
\begin{aligned}
& \nabla^{b}\left(\Delta^{k-1-a-b} u\right)(y) \cdot \nabla^{b}\left(\Delta^{a} \eta\right)(y) \\
& =\sum_{i_{1}, i_{2}, \ldots, i_{k-1}=1}^{n} \frac{\partial^{b}}{\partial y_{i_{1}} \partial y_{i_{2}} \cdots \partial y_{i_{b}}} \\
& \quad \times\left(\frac{\partial^{2}}{\partial y_{i_{1}}^{2}}\left(\frac{\partial^{2}}{\partial y_{i_{2}}^{2}}\left(\cdots\left(\frac{\partial^{2}}{\partial y_{i_{k-1-a-b}^{2}}^{2}} u\right) \cdots\right)\right)\right) \\
& \quad \frac{\partial^{b}}{\partial y_{i_{1}} \partial y_{i_{2}} \cdots \partial y_{i_{b}}}\left(\frac{\partial^{2}}{\partial y_{i_{1}}^{2}}\left(\frac{\partial^{2}}{\partial y_{i_{2}}^{2}}\left(\cdots\left(\frac{\partial^{2}}{\partial y_{i_{a}}^{2}} \eta\right) \cdots\right)\right)\right)
\end{aligned}
$$

and the summation is taken over all integers $a$ and $b$ satisfying $a \geq 0, b \geq 0$, and $0 \leq a+b \leq k-1$. Then, integrating by parts, for $x \in B_{R / 4}\left(x_{0}\right)$ we have

$$
\begin{aligned}
& u(x) \eta(x) \\
& =\int_{\mathbb{R}^{n}} \Gamma_{H_{k-1}, 0}(x, y)(-\Delta)^{k-1}(u \eta)(y) d y \\
& =\int_{\mathbb{R}^{n}} \Gamma_{H_{k-1}, 0}(x, y) \\
& \quad \times \sum_{a, b}\left\{(-1)^{k-1}\left(\begin{array}{c}
2(k-1) \\
2 a+b
\end{array}\right) \nabla^{b}\left(\Delta^{k-1-a-b} u\right)(y)\right. \\
& =\int_{\mathbb{R}^{n}} \Gamma_{H_{k-1}, 0}(x, y)(-\Delta)^{k-1} u(y) \eta(y) d y \\
& \quad+C \int_{\mathbb{R}^{n}} \sum_{(a, b) \neq(0,0)}\left\{\nabla^{b}\left(\Delta^{k-1-a-b} \Gamma_{H_{k-1}, 0}(x, y)\right)\right. \\
& \left.\cdot \nabla^{b}\left(\Delta^{a} \eta(y)\right) u(y)\right\} d y .
\end{aligned}
$$

Let

$$
\begin{aligned}
\left|u\left(x_{0}\right)\right| \leq & \int_{B_{R / 4}\left(x_{0}\right)} \frac{\left|(-\Delta)^{k-1} u(y)\right|}{\left|x_{0}-y\right|^{n-2(k-1)}} d y \\
& +\frac{C}{R^{2 a+b}} \sum_{(a, b) \neq(0,0)} \int_{B_{R / 4}\left(x_{0}\right) \backslash B_{R / 8}\left(x_{0}\right)} \frac{|u(y)|}{\left|x_{0}-y\right|^{n-2 a-b}} d y \\
& =I_{1}+I_{2} .
\end{aligned}
$$

Then we have

$$
\begin{aligned}
I_{2} & \leq \frac{C}{R^{n}} \int_{B_{R / 4}\left(x_{0}\right)}|u(y)| d y \\
& \leq C\left(\frac{1}{\left|B_{R}\left(x_{0}\right)\right|} \int_{B_{R}\left(x_{0}\right)}|u(y)|^{2} d y\right)^{1 / 2} .
\end{aligned}
$$

It remains to estimate $I_{1}$. Since $u \geq 0$, it follows that $\Delta\left((-\Delta)^{k-1} u\right)=-(-\Delta)^{k} u=V^{k} u \geq 0$. Hence $(-\Delta)^{k-1} u$ is subharmonic. Then for all $x \in B_{R / 4}\left(x_{0}\right)$,

$$
\begin{aligned}
(-\Delta)^{k-1} u(x) & \leq \frac{1}{\left|B_{R / 4}(x)\right|} \int_{B_{R / 4}(x)}(-\Delta)^{k-1} u(y) d y \\
& \leq C\left(\frac{1}{\left|B_{R / 2}\left(x_{0}\right)\right|} \int_{B_{R / 2}\left(x_{0}\right)}\left|\Delta^{k-1} u(y)\right|^{2} d y\right)^{1 / 2} .
\end{aligned}
$$

Then we have

$$
\begin{aligned}
I_{1} & \leq C \sup _{y \in B_{R / 4}\left(x_{0}\right)}\left|(-\Delta)^{k-1} u(y)\right| \int_{B_{R / 4}\left(x_{0}\right)} \frac{d y}{\left|x_{0}-y\right|^{n-2(k-1)}} \\
& \leq C R^{2(k-1)} \sup _{y \in B_{R / 4}\left(x_{0}\right)}\left|\Delta^{k-1} u(y)\right| \\
& \leq C R^{2 k-2}\left(\frac{1}{\left|B_{R / 2}\left(x_{0}\right)\right|} \int_{B_{R / 2}\left(x_{0}\right)}\left|\Delta^{k-1} u(y)\right|^{2} d y\right)^{1 / 2} .
\end{aligned}
$$

Then using Lemmas 13 and 15 repeatedly, we have

$$
\begin{array}{r}
I_{1} \leq C R^{2 k-2-n / 2}\left(\frac{1}{R^{2}} \int_{B_{R}\left(x_{0}\right)}\left|\nabla\left(\Delta^{k-2} u\right)(x)\right|^{2} d x\right. \\
\left.+\frac{1}{R^{4}} \int_{B_{R}\left(x_{0}\right)}\left|\Delta^{k-2} u(x)\right|^{2} d x\right)^{1 / 2} \\
\leq \cdots \leq C \sum_{j=0}^{k-1} R^{j}\left(\frac{1}{R^{n}} \int_{B_{R}\left(x_{0}\right)}\left|\nabla^{j} u(x)\right|^{2} d x\right)^{1 / 2} .
\end{array}
$$

It follows that

$$
\left|u\left(x_{0}\right)\right| \leq C \sum_{j=0}^{k-1} R^{j}\left(\frac{1}{\left|B_{R}\left(x_{0}\right)\right|} \int_{B_{R}\left(x_{0}\right)}\left|\nabla^{j} u(x)\right|^{2} d x\right)^{1 / 2} .
$$

From (26) we have, for all $y \in B_{R / 2}\left(x_{0}\right)$,

$$
|u(y)| \leq C \sum_{j=0}^{k-1} R^{j}\left(\frac{1}{\left|B_{R / 4}(y)\right|} \int_{B_{R / 4}(y)}\left|\nabla^{j} u(x)\right|^{2} d x\right)^{1 / 2} .
$$

Then we arrive at the desired estimate.

Lemma 18. Let $j, k$, and $n$ be integers, $k \geq 1, n \geq 2 k+1$, and $1 \leq j \leq 2 k-1$. Suppose that $V \in(R H)_{q_{0}}$ for some $q_{0}$, $n / 2 \leq q_{0}<k n /(2 k-j)$. Assume also that $(-\Delta)^{k} u+V^{k} u=0$ in 
$B_{R}\left(x_{0}\right)$ for some $x_{0} \in \mathbf{R}^{n}$. Then there exists a positive constant $C$ such that

$$
\begin{aligned}
& \left(\int_{B_{R / 2}\left(x_{0}\right)}\left|\nabla^{j} u(x)\right|^{t} d x\right)^{1 / t} \\
& \quad \leq C R^{k n / q_{0}-2 k}\left\{1+R \rho\left(x_{0}, V\right)\right\}^{2 k} \sup _{y \in B_{R}\left(x_{0}\right)}|u(y)|,
\end{aligned}
$$

where $1 / t=k / q_{0}-(2 k-j) / n$.

In Lemma 18, the cases $k=1$ and $k=2$ were shown in [3, Lemma 4.6] and [8, Lemma 7], respectively.

Proof of Lemma 18. We show Lemma 18 by a method similar to the one used in the proof of [3, Lemma 4.6]. Let $\eta \in$ $C_{0}^{\infty}\left(B_{R}\left(x_{0}\right)\right)$ such that $\eta \equiv 1$ on $B_{3 R / 4}\left(x_{0}\right)$ and $\left|\nabla^{b}\left(\Delta^{a} \eta\right)\right| \leq$ $C / R^{2 a+b}$, where $a$ and $b$ are nonnegative integers. We denote by $\Gamma_{H_{k}, 0}(x, y)$ the fundamental solution for $(-\Delta)^{k}$. Note that

$$
\begin{aligned}
& u(x) \eta(x) \\
& =\int_{\mathbf{R}^{n}} \Gamma_{H_{k}, 0}(x, y)\left\{-V(y)^{k} u(y) \eta(y)+(-1)^{k}\right. \\
& \times \sum_{a, b}\left(\begin{array}{c}
2 k \\
2 a+b
\end{array}\right) \nabla^{b}\left(\Delta^{k-a-b} u\right)(y) \\
& \left.\cdot \nabla^{b}\left(\Delta^{a} \eta\right)(y)\right\} d y,
\end{aligned}
$$

where the summation is taken over all integers $a$ and $b$ satisfying $a \geq 0, b \geq 0$, and $1 \leq a+b \leq k$. Then integrating by parts, for $x \in B_{R / 2}\left(x_{0}\right)$, we have

$$
\begin{aligned}
& \left|\nabla^{j} u(x)\right| \\
& \leq C \int_{B_{R}\left(x_{0}\right)} \frac{V(y)^{k}|u(y)||\eta(y)|}{|x-y|^{n-2 k+j}} d y \\
& \quad+\frac{C}{R^{n-2 k+j+b+2(k-a-b)+2 a+b}} \int_{B_{R}\left(x_{0}\right)}|u(y)| d y \\
& \leq C \sup _{y \in B_{R}\left(x_{0}\right)}|u(y)| \cdot \int_{B_{R}\left(x_{0}\right)} \frac{V(y)^{k}}{|x-y|^{n-2 k+j}} d y \\
& \quad+\frac{C}{R^{n+j}} \int_{B_{R}\left(x_{0}\right)}|u(y)| d y .
\end{aligned}
$$

It then follows from the well-known theorem on fractional integrals that

$$
\begin{aligned}
& \left(\int_{B_{R / 2}\left(x_{0}\right)}\left|\nabla^{j} u(x)\right|^{t} d x\right)^{1 / t} \\
& \quad \leq C \sup _{y \in B_{R}\left(x_{0}\right)}|u(y)|\left(\int_{B_{R}\left(x_{0}\right)} V(x)^{q_{0}} d x\right)^{k / q_{0}}
\end{aligned}
$$

$$
\begin{aligned}
& +C R^{k n / q_{0}-2 k} \sup _{y \in B_{R}\left(x_{0}\right)}|u(y)| \\
\leq & C R^{k n / q_{0}-2 k}\left\{1+R \rho\left(x_{0}, V\right)\right\}^{2 k} \sup _{y \in B_{R}\left(x_{0}\right)}|u(y)|,
\end{aligned}
$$

where $1 / t=k / q_{0}-(2 k-j) / n$ and we have used Remark 4 (1).

At the end of this section, we give the following.

Proof of Lemma 13. Let $l$ and $m$ be nonnegative integers and choose $\eta \in C_{0}^{\infty}\left(B_{R}\left(x_{0}\right)\right)$ such that $\eta \equiv 1$ on $B_{R / 2}\left(x_{0}\right)$ and $\left|\nabla^{m}\left(\Delta^{l} \eta\right)\right| \leq C / R^{2 l+m}$. Multiplying $(-\Delta)^{k} u+V^{k} u=0$ by $\overline{\left((-\Delta)^{k-2 a} u\right)} \eta^{k+1}$ and integrating over $\mathbf{R}^{n}$ by integrating by parts, we have

$$
\begin{aligned}
& \int_{B_{R / 2}\left(x_{0}\right)}\left|\Delta^{k-a} u(x)\right|^{2} d x \\
& \quad+\int_{B_{R / 2}\left(x_{0}\right)} V(x)^{k}|u(x)|\left|\Delta^{k-2 a} u(x)\right| d x \\
& \leq C \int_{B_{R}\left(x_{0}\right)} \sum_{l, m}\left|\Delta^{k-a} u(x)\right| \mid \nabla^{m}\left(\Delta^{k-a-l-m} u\right)(x) \\
& \cdot \nabla^{m}\left(\Delta^{l} \eta^{k+1}\right)(x) \mid d x,
\end{aligned}
$$

where the summation is taken over all integers $l$ and $m$ satisfying $l \geq 0, m \geq 0$, and $1 \leq l+m \leq a$. Let $\epsilon$ be a positive real number which will be determined later. Then the righthand side of (32) is bounded by

$$
\begin{gathered}
C \int_{B_{R}\left(x_{0}\right)} \sum_{l, m} \sqrt{\epsilon}\left|\Delta^{k-a} u(x)\right| \cdot \frac{1}{\sqrt{\epsilon}} \\
\cdot \frac{1}{R^{2 l+m}}\left|\nabla^{m}\left(\Delta^{k-a-l-m} u\right)(x)\right| d x \\
\leq C \int_{B_{R}\left(x_{0}\right)} \sum_{l, m}\left(\epsilon\left|\Delta^{k-a} u(x)\right|^{2}+\frac{1}{\epsilon} \cdot \frac{1}{R^{2(2 l+m)}}\right. \\
\left.\times\left|\nabla^{m}\left(\Delta^{k-a-l-m} u\right)(x)\right|^{2}\right) d x .
\end{gathered}
$$

Then choosing $\epsilon$ such that $C(a-1)(a+2) \epsilon=1$, we arrive at the desired estimate.

\section{Estimates for $H_{2^{l}}$}

In this section, we assume that Theorem 9 is true for $m=l$ and show estimates for $\mathrm{H}_{2^{l}}$ which is needed to prove the case $m=l+1$ in Theorem 9 .

Lemma 19. Let $j, l$, and $n$ be integers, $l \geq 0$, and $n \geq 2^{l+1}+1$. Suppose that $V \in(R H)_{n / 2}$. Assume also that Theorem 9 is true for $m=l$. Then there exists a positive constant $C$ such that

$$
\left\|\rho(\cdot, V)^{2^{l+1}} H_{2^{l}}^{-1} f\right\|_{L^{p}\left(\mathbf{R}^{n}\right)} \leq C\|f\|_{L^{p}\left(\mathbf{R}^{n}\right)},
$$

where $1 \leq p \leq \infty$. 
Proof. We can prove Lemma 19 by the same way as in the proof of [3, Corollary 2.8]. It follows from the inductive assumption that

$$
\begin{aligned}
& \int_{\mathbf{R}^{n}}\left|\Gamma_{H_{2^{l}}}(x, y)\right| d y \\
& \quad \leq C \int_{\mathbf{R}^{n}} \frac{d y}{\{1+\rho(x, V)|x-y|\}^{2^{l+1}+1}|x-y|^{n-2^{l+1}}} \\
& =\frac{C}{\rho(x, V)^{2^{l+1}}} .
\end{aligned}
$$

If $f \in L^{p}\left(\mathbf{R}^{n}\right)$, then

$$
u(x)=H_{2^{l}}^{-1} f(x)=\int_{\mathbf{R}^{n}} \Gamma_{H_{2^{l}}}(x, y) f(y) d y .
$$

Let $p^{\prime}=p /(p-1)$. Then using Hölder's inequality and (35) we have

$$
\begin{aligned}
|u(x)| \leq & \left(\int_{\mathrm{R}^{n}}\left|\Gamma_{H_{2^{l}}}(x, y)\right| d y\right)^{1 / p^{\prime}} \\
& \times\left(\int_{\mathrm{R}^{n}}\left|\Gamma_{H_{2^{l}}}(x, y)\right||f(y)|^{p} d y\right)^{1 / p} \\
\leq & \frac{C}{\rho(x, V)^{2^{l+1} / p^{\prime}}}\left(\int_{\mathrm{R}^{n}}\left|\Gamma_{H_{2^{l}}}(x, y)\right||f(y)|^{p} d y\right)^{1 / p} .
\end{aligned}
$$

It follows that

$$
\begin{aligned}
& \int_{\mathbf{R}^{n}}\left|\rho(x, V)^{2^{l+1}} u(x)\right|^{p} d x \\
& \quad \leq C \int_{\mathbf{R}^{n}}|f(y)|^{p}\left(\int_{\mathbf{R}^{n}} \rho(x, V)^{2^{l+1}}\left|\Gamma_{H_{2^{l}}}(x, y)\right| d x\right) d y .
\end{aligned}
$$

Finally, note that, by the inductive assumption and Lemma 12 (1),

$$
\begin{aligned}
& \int_{\mathbf{R}^{n}} \rho(x, V)^{2^{l+1}}\left|\Gamma_{H_{2^{l}}}(x, y)\right| d x \\
& \quad \leq C C_{N} \int_{\mathbf{R}^{n}} \frac{\rho(y, V)^{2^{l+1}}}{\{1+\rho(y, V)|x-y|\}^{N-2^{l+1} k_{0}}|x-y|^{n-2^{l+1}}} d x \\
& \quad \leq C
\end{aligned}
$$

if we choose $N=2^{l+1} k_{0}+2^{l+1}+1$. Then Lemma 19 follows.

We also need the following lemma.

Lemma 20. Let $j, l$, and $n$ be integers, $l \geq 0, n \geq 2^{l+1}+1$, and $1 \leq j \leq 2^{l+1}-1$. Suppose that $V \in(R H)_{q_{0}}$ for some $q_{0}$, $n / 2 \leq q_{0}<2^{l} n /\left(2^{l+1}-j\right)$. Assume also that Theorem 9 is true for $m=l$. Then for $1 \leq p \leq p_{0}$ there exists a positive constant C such that

$$
\left\|\rho(\cdot, V)^{2^{l+1}-j} \nabla^{j} H_{2^{l}}^{-1} f\right\|_{L^{p}\left(\mathbf{R}^{n}\right)} \leq C\|f\|_{L^{p}\left(\mathbf{R}^{n}\right)}
$$

where $1 / p_{0}=2^{l} / q_{0}-\left(2^{l+1}-j\right) / n$.

Proof. We show Lemma 20 by a method similar to the one used in the proof of [3, Theorem 4.13]. Suppose that $V \in$ $(R H)_{q_{0}}$ for some $q_{0} \geq n / 2$. Then $V \in(R H)_{q_{1}}$ for some $q_{1}$, $2^{l} n /\left(2^{l+1}-j\right)>q_{1}>q_{0}$. Let

$$
T_{2^{l}} f(x)=\rho(x, V)^{2^{l+1}-j} \int_{\mathbf{R}^{n}} \nabla_{x}^{j} \Gamma_{H_{2^{l}}}(x, y) f(y) d y .
$$

The adjoint of $T_{2^{l}}$ is given by

$$
T_{2^{l}}^{*} f(x)=\int_{\mathbf{R}^{n}} \nabla_{y}^{j} \Gamma_{H_{2^{l}}}(y, x) \rho(y, V)^{2^{l+1}-j} f(y) d y .
$$

By duality, it suffices to show that

$$
\left\|T_{2^{l}}^{*} f\right\|_{L^{p}\left(\mathbf{R}^{n}\right)} \leq C\|f\|_{L^{p}\left(\mathbf{R}^{n}\right)} \quad \text { for } p_{0}^{\prime} \leq p \leq \infty
$$

where $1 / p_{0}+1 / p_{0}^{\prime}=1$. Let $r=1 / \rho(x, V)$. We choose $t$ and $p_{1}$ such that $1 / t=2^{l} / q_{1}-\left(2^{l+1}-j\right) / n, 1 / p_{1}=1-2^{l} / q_{1}+\left(2^{l+1}-\right.$ $j) / n$. Thus $1 / t+1 / p_{1}=1$. By Hölder's inequality we have

$$
\begin{aligned}
& \left|T_{2^{l}}^{*} f(x)\right| \\
& \leq \sum_{i=-\infty}^{\infty} \int_{2^{i-1} r<|y-x| \leq 2^{i_{r}}}\left|\nabla_{y}^{j} \Gamma_{H_{2^{l}}}(y, x)\right| \\
& \quad \times \rho(y, V)^{2^{l+1}-j}|f(y)| d y \\
& \leq \sum_{i=-\infty}^{\infty}\left(\int _ { 2 ^ { i ^ { i - 1 } r < | y - x | \leq 2 ^ { i } r } } \left\{\left|\nabla_{y}^{j} \Gamma_{H_{2^{l}}}(y, x)\right|\right.\right. \\
& \left.\left.\times \rho(y, V)^{2^{l+1}-j}\right\}^{t} d y\right)^{1 / t} \\
& \quad \cdot\left(\int_{|y-x| \leq 2^{i_{r}}}|f(y)|^{p_{1}} d y\right)^{1 / p_{1}} .
\end{aligned}
$$


Let $a$ be a positive integer and $y_{a} \in \mathbf{R}^{n}$. It follows from Lemma 18, Lemma 12 (1), and the inductive assumption that

$$
\begin{aligned}
& \left(\int_{2^{i-1} r<|y-x| \leq 2^{i} r}\left\{\left|\nabla_{y}^{j} \Gamma_{H_{2^{l}}}(y, x)\right| \rho(y, V)^{2^{l+1}-j}\right\}^{t} d y\right)^{1 / t} \\
& \leq \sum_{a=1}^{A}\left(\int_{\left|z-y_{a}\right| \leq 2^{i-1} r}\left\{\left|\nabla_{z}^{j} \Gamma_{H_{2^{l}}}(z, x)\right| \rho(z, V)^{2^{l+1}-j}\right\}^{t} d z\right)^{1 / t} \\
& \leq C\left(2^{i-1} r\right)^{2^{l} n / q_{1}-2^{l+1}}\left\{1+2^{i} r \rho(x, V)\right\}^{2^{l+1}+\left(2^{l+1}-j\right) k_{0}} \\
& \quad \times \rho(x, V)^{2^{l+1}-j} \cdot \sum_{a=1}^{A} \sup _{z \in B_{5, i^{i-3} r}\left(y_{a}\right)}\left|\Gamma_{H_{2^{l}}}(z, x)\right| \\
& \leq C\left(2^{i} r\right)^{2^{l} n / q_{1}-2^{l+1}}\left(1+2^{i}\right)^{2^{l+1}+\left(2^{l+1}-j\right) k_{0}} \cdot \frac{1}{r^{2^{l+1}-j}} \\
& \quad \cdot \frac{C_{N}}{\{1+\rho(x, V)|z-x|\}^{N}} \cdot \sum_{a=1}^{A} \sup _{z \in B_{5,2^{2}-3} r}\left(y_{a}\right)|z-x|^{n-2^{l+1}} \\
& \leq C C_{N} \frac{\left(2^{i} r\right)^{2^{l} n / q_{1}-2^{l+1}}\left(1+2^{i}\right)^{2^{l+1}+\left(2^{l+1}-j\right) k_{0}}}{r^{2^{l+1}-j}\left(1+2^{i-3}\right)^{N}\left(2^{i-3} r\right)^{n-2^{l+1}}} \\
& \leq C C_{N} \frac{\left(2^{i} r\right)^{2^{l} n / q_{1}-n}}{r^{2^{l+1}-j}\left(1+2^{i}\right)^{N-2^{l+1}-\left(2^{l+1}-j\right) k_{0}},}
\end{aligned}
$$

where $A$ is a finite integer not depending on $i$ and $r$. Thus

$$
\begin{aligned}
\left|T_{2^{l}}^{*} f(x)\right| \leq C C_{N} \sum_{i=-\infty}^{\infty} \frac{\left(2^{i}\right)^{2^{l+1}-j}}{\left(1+2^{i}\right)^{N-2^{l+1}-\left(2^{l+1}-j\right) k_{0}}} \\
\quad \times\left\{\frac{1}{\left(2^{i} r\right)^{n}} \int_{B_{2^{i}}(x)}|f(y)|^{p_{1}} d y\right\}^{1 / p_{1}} \\
\leq C\left\{M\left(|f|^{p_{1}}\right)(x)\right\}^{1 / p_{1}},
\end{aligned}
$$

where we choose $N \geq 2^{l+2}+\left(2^{l+1}-j\right) k_{0}-j+1$ and $M$ is the Hardy-Littlewood maximal operator. Hence it follows that

$$
\left\|T_{2^{l}}^{*} f\right\|_{L^{p}\left(\mathbf{R}^{n}\right)} \leq C\|f\|_{L^{p}\left(\mathbf{R}^{n}\right)} \text { for } p_{1}<p \leq \infty .
$$

Then (43) follows since $p_{0}^{\prime}>p_{1}$.

For the case $q_{0}=n / 2$ in Lemma 20 , if $n \geq 2^{l+2}+1$, letting $p=2$, we have the following.

Corollary 21. Let $j, l$, and $n$ be integers, $l \geq 0, n \geq 2^{l+2}+1$, and $1 \leq j \leq 2^{l+1}-1$. Suppose that $V \in(R H)_{n / 2}$. Assume also that Theorem 9 is true for $m=l$. Then there exists a positive constant $C$ such that

$$
\left\|\rho(\cdot, V)^{2^{l+1}-j} \nabla^{j} H_{2^{l}}^{-1} f\right\|_{L^{2}\left(\mathbf{R}^{n}\right)} \leq C\|f\|_{L^{2}\left(\mathbf{R}^{n}\right)} .
$$

\section{Proof of Theorem 9 (Case $m=l+1)$}

In this section, we assume that Theorem 9 is true for $m=l$ and prove the case $m=l+1$ in Theorem 9. It follows easily from the following lemma.

Lemma 22. Let $j, l$, and $n$ be integers, $l \geq 0$, and $n \geq 2^{l+2}+$ 1. Suppose that $V \in(R H)_{n / 2}$. Assume also that $(-\Delta)^{2^{l+1}} u+$ $V^{2^{l+1}} u=0$ in $B_{R}\left(x_{0}\right)$ for some $x_{0} \in \mathbf{R}^{n}$ and Theorem 9 is true for $m=l$. Then for any positive integer $N$ there exists a positive constant $C_{N}$ such that

$$
\sup _{y \in B_{R / 2}\left(x_{0}\right)}|u(y)| \leq \frac{C_{N}}{\left\{1+R \rho\left(x_{0}, V\right)\right\}^{N}} \sup _{y \in B_{R}\left(x_{0}\right)}|u(y)| .
$$

Assuming Lemma 22 for the moment, we give

Proof of Theorem 9 (Case $m=l+1$ ). Fix $x_{0}, y_{0} \in \mathbf{R}^{n}$ and put $R=\left|x_{0}-y_{0}\right|$. Then $u(x)=\Gamma_{H_{2} l+1}\left(x, y_{0}\right)$ is a solution of $(-\Delta)^{2^{l+1}} u+V^{2^{l+1}} u=0$ on $B_{R / 2}\left(x_{0}\right)$. Using the estimate $0 \leq \Gamma_{H_{2+1}}(x, y) \leq C|x-y|^{l^{2+2}-n}$ and (49), we arrive at the desired estimate.

To prove Lemma 22, we need some lemmas.

Lemma 23. Let $j, l$, and $n$ be integers, $l \geq 0, n \geq 2^{l+2}+1$, and $0 \leq j \leq 2^{l+1}-1$. Suppose that $V \in(R H)_{n / 2}$. Assume also that Theorem 9 is true for $m=l$. Then there exists a positive constant $C$ such that

$$
\begin{aligned}
& \sum_{j=0}^{2^{l+1}-1} \int_{\mathbf{R}^{n}} \rho(x, V)^{2\left(2^{l+1}-j\right)}\left|\nabla^{j} u(x)\right|^{2} d x \\
& \quad \leq C\left(\int_{\mathbf{R}^{n}}\left|\Delta^{2^{l}} u(x)\right|^{2} d x+\int_{\mathbf{R}^{n}} V(x)^{2^{l+1}}|u(x)|^{2} d x\right),
\end{aligned}
$$

where $u \in C_{0}^{\infty}\left(\mathbf{R}^{n}\right)$.

Proof. By the case $p=2$ in Lemma 19 and Corollary 21, we have

$$
\begin{aligned}
& \sum_{j=0}^{2^{l+1}-1} \int_{\mathbf{R}^{n}} \rho(x, V)^{2\left(2^{l+1}-j\right)}\left|\nabla^{j} u(x)\right|^{2} d x \\
& \quad \leq C \int_{\mathbf{R}^{n}}\left|\left\{(-\Delta)^{2^{l}}+V^{2^{l}}\right\} u(x)\right|^{2} d x \\
& \quad \leq C\left(\int_{\mathbf{R}^{n}}\left|\Delta^{2^{l}} u(x)\right|^{2} d x+\int_{\mathbf{R}^{n}} V(x)^{2^{l+1}}|u(x)|^{2} d x\right) .
\end{aligned}
$$

If $n \geq 2^{l+2}+1$ and $1 \leq j \leq 2^{l+1}-1$, then letting $k=2^{l+1}$ and $q_{0}=n / 2$ in Lemma 18 we have the following. 
Corollary 24. Let $j, l$, and $n$ be integers, $l \geq 0, n \geq 2^{l+2}+1$, and $1 \leq j \leq 2^{l+1}-1$. Suppose that $V \in(R H)_{n / 2}$. Assume also that $(-\Delta)^{2^{l+1}} u+V^{2^{2+1}} u=0$ in $B_{R}\left(x_{0}\right)$ for some $x_{0} \in \mathbf{R}^{n}$. Then there exists a positive constant $C$ such that

$$
\begin{aligned}
& \left(\frac{1}{\left|B_{R / 2}\left(x_{0}\right)\right|} \int_{B_{R / 2}\left(x_{0}\right)}\left|\nabla^{j} u(x)\right|^{2} d x\right)^{1 / 2} \\
& \leq \frac{C\left\{1+R \rho\left(x_{0}, V\right)\right\}^{2^{l+2}}}{R^{j}} \sup _{y \in B_{R}\left(x_{0}\right)}|u(y)| .
\end{aligned}
$$

Now we are ready to give the following.

Proof of Lemma 22. Let $\eta \in C_{0}^{\infty}\left(B_{R / 2}\left(x_{0}\right)\right)$ such that $\eta \equiv 1$ on $B_{R / 4}\left(x_{0}\right),\left|\nabla^{j} \eta\right| \leq C / R^{j}$, where $j$ is an integer satisfying $1 \leq$ $j \leq 2^{l+1}-1$. Applying Lemma 23 to $u \eta$ and using Corollary 14 we have

$$
\begin{aligned}
& \sum_{j=0}^{2^{l+1}-1} \int_{B_{R / 4}\left(x_{0}\right)} \rho(x, V)^{2\left(2^{l+1}-j\right)}\left|\nabla^{j} u(x)\right|^{2} d x \\
& \quad \leq C \sum_{j=0}^{2^{l+1}-1} \frac{1}{R^{2\left(2^{l+1}-j\right)}} \int_{B_{R}\left(x_{0}\right)}\left|\nabla^{j} u(x)\right|^{2} d x .
\end{aligned}
$$

From Lemma 12 (2) it follows for each integer $j$ satisfying $1 \leq$ $j \leq 2^{l+1}-1$ that

$$
\begin{aligned}
\int_{B_{R / 4}\left(x_{0}\right)}\left|\nabla^{j} u(x)\right|^{2} d x \\
\leq \frac{C\left\{1+R \rho\left(x_{0}, V\right)\right\}^{2\left(2^{l+1}-j\right) k_{0} /\left(k_{0}+1\right)}}{\left\{R \rho\left(x_{0}, V\right)\right\}^{2\left(2^{l+1}-j\right)}} \cdot \frac{R^{2^{l+2}}}{R^{2 j}} \\
\quad \cdot \sum_{i=0}^{2^{l+1}-1} \frac{R^{2 i}}{R^{2^{l+2}}} \int_{B_{R}\left(x_{0}\right)}\left|\nabla^{i} u(x)\right|^{2} d x .
\end{aligned}
$$

Then we have

$$
\begin{gathered}
\sum_{j=0}^{2^{l+1}-1} R^{j}\left(\frac{1}{\left|B_{R / 4}\left(x_{0}\right)\right|} \int_{B_{R / 4}\left(x_{0}\right)}\left|\nabla^{j} u(x)\right|^{2} d x\right)^{1 / 2} \\
\leq \frac{C}{\left\{1+R \rho\left(x_{0}, V\right)\right\}^{1 /\left(k_{0}+1\right)}} \sum_{i=0}^{2^{l+1}-1} R^{i} \\
\cdot\left(\frac{1}{\left|B_{R}\left(x_{0}\right)\right|} \int_{B_{R}\left(x_{0}\right)}\left|\nabla^{i} u(x)\right|^{2} d x\right)^{1 / 2} \cdot
\end{gathered}
$$

Repeating the above argument, for any positive integer $K$ we have

$$
\sum_{j=0}^{2^{l+1}-1} R^{j}\left(\frac{1}{\left|B_{R / 4^{K}}\left(x_{0}\right)\right|} \int_{B_{R / 4^{K}}\left(x_{0}\right)}\left|\nabla^{j} u(x)\right|^{2} d x\right)^{1 / 2}
$$

$$
\begin{aligned}
\leq & \frac{C_{K}}{\left\{1+R \rho\left(x_{0}, V\right)\right\}^{K /\left(k_{0}+1\right)}} \sum_{j=0}^{2^{l+1}-1} R^{j} \\
& \cdot\left(\frac{1}{\left|B_{R}\left(x_{0}\right)\right|} \int_{B_{R}\left(x_{0}\right)}\left|\nabla^{j} u(x)\right|^{2} d x\right)^{1 / 2} .
\end{aligned}
$$

Then using Lemma 17, Corollary 24, and estimate (56), we arrive at the desired estimate.

\section{Proof of Theorem 10}

In this section, we prove Theorem 10 which states derivative estimates of the fundamental solution for $H_{2}$. We arrive at Theorem 10 combining Lemmas 25 with 22.

Lemma 25. Let $j, m$, and $n$ be integers, $m \geq 0, n \geq 2^{m+1}+1$, and $1 \leq j \leq 2^{m+1}-1$. Suppose that $V \in(R H)_{2^{m} n /\left(2^{m+1}-j\right)}$. Assume also that $(-\Delta)^{2^{m}} u+V^{2^{m}} u=0$ in $B_{R}\left(x_{0}\right)$ for some $x_{0} \epsilon$ $\mathbf{R}^{n}$. Then there exist positive constants $C_{j}$ and $C_{j}^{\prime}$ such that

$$
\sup _{y \in B_{R / 2}\left(x_{0}\right)}\left|\nabla^{j} u(y)\right| \leq \frac{C_{j}\left\{1+R \rho\left(x_{0}, V\right)\right\}^{C_{j}^{\prime}}}{R^{j}} \sup _{y \in B_{R}\left(x_{0}\right)}|u(y)| .
$$

Proof. Let $\eta \in C_{0}^{\infty}\left(B_{R}\left(x_{0}\right)\right)$ such that $\eta \equiv 1$ on $B_{3 R / 4}\left(x_{0}\right)$ and $\left|\nabla^{b}\left(\Delta^{a} \eta\right)\right| \leq C / R^{2 a+b}$, where $a$ and $b$ are nonnegative integers. We use (29); integrating by parts, we have

$$
\begin{aligned}
\left|\nabla^{j} u\left(x_{0}\right)\right| \leq C & \int_{B_{R}\left(x_{0}\right)} \frac{V(y)^{2^{m}}|u(y)|}{\left|x_{0}-y\right|^{n-2^{m+1}+j}} d y \\
& +\frac{C}{R^{n+j}} \int_{B_{R}\left(x_{0}\right)}|u(y)| d y .
\end{aligned}
$$

Since $V \in(R H)_{2^{m} n /\left(2^{m+1}-j\right)}$, it follows that $V \in(R H)_{q}$ for some $q>2^{m} n /\left(2^{m+1}-j\right)$. We choose $r$ such that $2^{m} / q+1 / r=1$ and $r>1$. By Hölder's inequality we have

$$
\begin{aligned}
\left|\nabla^{j} u\left(x_{0}\right)\right| & \\
\leq & C R^{n}\left(\frac{1}{R^{n}} \int_{B_{R}\left(x_{0}\right)} V(y)^{q} d y\right)^{2^{m} / q} \\
& \cdot\left(\frac{1}{R^{n}} \int_{B_{R}\left(x_{0}\right)} \frac{d y}{\left|x_{0}-y\right|^{\left(n-2^{m+1}+j\right) r}}\right)^{1 / r} \sup _{y \in B_{R}\left(x_{0}\right)}|u(y)| \\
& +\frac{C}{R^{n+j}} \int_{B_{R}\left(x_{0}\right)}|u(y)| d y \\
\leq & \frac{C\left\{1+R \rho\left(x_{0}, V\right)\right\}^{2^{m+1}}}{R^{j}} \sup _{y \in B_{R}\left(x_{0}\right)}|u(y)|,
\end{aligned}
$$


where we have used Remark 4 (1). From (59) we have, for all $y \in B_{R / 2}\left(x_{0}\right)$,

$$
\left|\nabla^{j} u(y)\right| \leq \frac{C\{1+R \rho(y, V)\}^{2^{m+1}}}{R^{j}} \sup _{x \in B_{R / 4}(y)}|u(x)| .
$$

Using Lemma 12 (1), we have

$$
\begin{aligned}
& \sup _{y \in B_{R / 2}\left(x_{0}\right)}\left|\nabla^{j} u(y)\right| \\
& \leq \frac{C\left\{1+R \rho\left(x_{0}, V\right)\right\}^{2^{m+1}\left(k_{0}+1\right)}}{R^{j}} \sup _{y \in B_{R}\left(x_{0}\right)}|u(y)| .
\end{aligned}
$$

Then the proof is complete.

\section{Proofs of Theorems 5 and 8}

In this section, we prove Theorems 5 and 8 .

Proof of Theorem 5. We assume that $V \in(R H)_{q_{0}}$ for some $q_{0} \geq 2^{m} n /\left(2^{m+1}-j\right)$. Then $V \in(R H)_{q_{1}}$ for some $q_{1}>q_{0}$. Let

$$
T_{2^{m}} f(x)=V(x)^{\left(2^{m+1}-j\right) / 2} \int_{\mathbf{R}^{n}} \nabla_{x}^{j} \Gamma_{H_{2^{m}}}(x, y) f(y) d y .
$$

The adjoint of $T_{2^{m}}$ is given by

$$
T_{2^{m}}^{*} f(x)=\int_{\mathbf{R}^{n}} \nabla_{y}^{j} \Gamma_{H_{2^{m}}}(y, x) V(y)^{\left(2^{m+1}-j\right) / 2} f(y) d y .
$$

Let $p_{0}=2 q_{0} /\left(2^{m+1}-j\right)$. By duality, it suffices to show that

$$
\left\|T_{2^{m}}^{*} f\right\|_{L^{p}\left(\mathbf{R}^{n}\right)} \leq C\|f\|_{L^{p}\left(\mathbf{R}^{n}\right)} \quad \text { for } p_{0}^{\prime} \leq p \leq \infty
$$

where $1 / p_{0}+1 / p_{0}^{\prime}=1$. Let $r=1 / \rho(x, V)$. By Theorems 9 and 10 and Hölder's inequality we have

$$
\begin{aligned}
& \left|T_{2^{m}}^{*} f(x)\right| \\
& \leq \sum_{i=-\infty}^{\infty} \int_{2^{i-1} r<|y-x| \leq 2^{i} r}\left|\nabla_{y}^{j} \Gamma_{H_{2^{m}}}(y, x)\right| \\
& \times V(y)^{\left(2^{m+1}-j\right) / 2}|f(y)| d y \\
& \leq C C_{N} \sum_{i=-\infty}^{\infty} \frac{1}{\left(1+2^{i}\right)^{N}} \cdot \frac{1}{\left(2^{i} r\right)^{n-2^{m+1}+j}} \\
& \times \int_{B_{2^{i} r}(x)} V(y)^{\left(2^{m+1}-j\right) / 2}|f(y)| d y \\
& \leq C C_{N} \sum_{i=-\infty}^{\infty} \frac{\left(2^{i} r\right)^{2^{m+1}-j}}{\left(1+2^{i}\right)^{N}} \\
& \quad \times\left\{\frac{1}{\left(2^{i} r\right)^{n}} \int_{B_{2^{i}}(x)} V(y)^{q_{1}} d y\right\}^{\left(2^{m+1}-j\right) /\left(2 q_{1}\right)} \\
& \quad\left\{\frac{1}{\left(2^{i} r\right)^{n}} \int_{B_{2^{i} r}(x)}|f(y)|^{p_{1}} d y\right\}^{1 / p_{1}},
\end{aligned}
$$

where $1 / p_{1}=1-\left(2^{m+1}-j\right) /\left(2 q_{1}\right)$. Since $V \in(R H)_{q_{1}}$ we have

$$
\begin{aligned}
& \left|T_{2^{m}}^{*} f(x)\right| \\
& \leq C C_{N}\left\{M\left(|f|^{p_{1}}\right)(x)\right\}^{1 / p_{1}} \\
& \quad \cdot \sum_{i=-\infty}^{\infty} \frac{1}{\left(1+2^{i}\right)^{N}}\left\{\frac{1}{\left(2^{i} r\right)^{n-2}} \int_{B_{2^{i}}(x)} V(y) d y\right\}^{\left(2^{m+1}-j\right) / 2} .
\end{aligned}
$$

For the case $j \leq 0$, by Lemma 11 and (4), we have

$$
\begin{aligned}
& \frac{1}{\left(2^{i} r\right)^{n-2}} \int_{B_{2^{i} r}(x)} V(y) d y \\
& \quad \leq C\left(\frac{r}{2^{i} r}\right)^{n / q_{1}-2} \cdot \frac{1}{r^{n-2}} \int_{B_{r}(x)} V(y) d y \\
& \quad=C\left(2^{i}\right)^{2-n / q_{1}} .
\end{aligned}
$$

For the case $j \geq 1$, by the doubling condition (9) and (4), we have

$$
\begin{aligned}
& \frac{1}{\left(2^{i} r\right)^{n-2}} \int_{B_{2^{i_{r}}}(x)} V(y) d y \\
& \quad \leq 2^{2 i} \cdot 2^{-i n} C_{0}^{i} \cdot \frac{1}{r^{n-2}} \int_{B_{r}(x)} V(y) d y \\
& \quad \leq C\left(2^{i}\right)^{k_{1}},
\end{aligned}
$$

where $k_{1}=2-n+\log _{2} C_{0}$. Hence, choosing $N \geq k_{1}\left(2^{m+1}-\right.$ j) $/ 2+1$, we obtain

$$
\left|T_{2^{m}}^{*} f(x)\right| \leq C\left\{M\left(|f|^{p_{1}}\right)(x)\right\}^{1 / p_{1}} .
$$

It follows that

$$
\left\|T_{2^{m}}^{*} f\right\|_{L^{p}\left(\mathbf{R}^{n}\right)} \leq C\|f\|_{L^{p}\left(\mathbf{R}^{n}\right)} \quad \text { for } p_{1}<p \leq \infty .
$$

Then (64) follows since $p_{1}=\left\{2 q_{1} /\left(2^{m+1}-j\right)\right\}^{\prime}<$ $\left\{2 q_{0} /\left(2^{m+1}-j\right)\right\}^{\prime}=p_{0}^{\prime}$.

Proof of Theorem 8. We assume that $V \in(R H)_{q_{0}}$ for some $q_{0}$, $n / 2 \leq q_{0}<2^{m} n /\left(2^{m+1}-j\right)$. Then $V \in(R H)_{q_{1}}$ for some $q_{1}$, $2^{m} n /\left(2^{m+1}-j\right)>q_{1}>q_{0}$. We use (62) and (63) again and it suffices to show $(64)$ for $1 / p_{0}=\left(2^{m+2}-j\right) /\left(2 q_{0}\right)-\left(2^{m+1}-j\right) / n$ and $1 / p_{0}+1 / p_{0}^{\prime}=1$. We choose $t$ and $p_{1}$ such that $1 / t=$ $2^{m} / q_{1}-\left(2^{m+1}-j\right) / n$ and $1 / p_{1}=1-\left(2^{m+2}-j\right) /\left(2 q_{1}\right)+\left(2^{m+1}-\right.$ j)/n. Thus,

$$
\frac{1}{t}+\frac{2^{m+1}-j}{2 q_{1}}+\frac{1}{p_{1}}=1 .
$$


Let $r=1 / \rho(x, V)$. By Hölder's inequality we have

$$
\begin{aligned}
& \left|T_{2^{m}}^{*} f(x)\right| \\
& \leq \sum_{i=-\infty}^{\infty}\left(\int_{2^{i-1} r<|y-x| \leq 2^{i} r}\left|\nabla_{y}^{j} \Gamma_{H_{2^{m}}}(y, x)\right|^{t} d y\right)^{1 / t} \\
& \quad \cdot\left(\int_{|y-x| \leq 2^{i} r} V(y)^{q_{1}} d y\right)^{\left(2^{m+1}-j\right) /\left(2 q_{1}\right)} \\
& \quad \times\left(\int_{|y-x| \leq 2^{i} r}|f(y)|^{p_{1}} d y\right)^{1 / p_{1}} .
\end{aligned}
$$

It follows from Lemma 18 and Theorem 9 that

$$
\begin{gathered}
\left(\int_{2^{i-1} r<|y-x| \leq 2^{i} r}\left|\nabla_{y}^{j} \Gamma_{H_{2} m}(y, x)\right|^{t} d y\right)^{1 / t} \\
\leq C_{N} \frac{\left(2^{i} r\right)^{2^{m} n / q_{1}-n}}{\left(1+2^{i}\right)^{N}} .
\end{gathered}
$$

Thus, as in the proof of Theorem 5, we have

$$
\begin{aligned}
& \left|T_{2^{m}}^{*} f(x)\right| \\
& \leq C C_{N}\left\{M\left(|f|^{p_{1}}\right)(x)\right\}^{1 / p_{1}} \\
& \quad \cdot \sum_{i=-\infty}^{\infty} \frac{1}{\left(1+2^{i}\right)^{N}}\left\{\frac{1}{\left(2^{i} r\right)^{n-2}} \int_{B_{2^{i_{r}}}(x)} V(y) d y\right\}^{\left(2^{m+1}-j\right) / 2} \\
& \leq C\left\{M\left(|f|^{p_{1}}\right)(x)\right\}^{1 / p_{1}} .
\end{aligned}
$$

Then (64) follows since $p_{0}^{\prime}>p_{1}$.

\section{Remarks}

We show estimate (57) under another assumption.

Lemma 26. Let $k, l$, and $n$ be integers, $m \geq 0, n \geq 2^{m+1}+1$, and $1 \leq j \leq 2^{m+1}-1$. Suppose that $V \in(R H)_{n / 2}$ and there exists a positive constant $C$ such that $V(x) \leq C \rho(x, V)^{2}$. Assume also that $(-\Delta)^{2^{m}} u+V^{2^{m}} u=0$ in $B_{R}\left(x_{0}\right)$ for some $x_{0} \in \mathbf{R}^{n}$. Then there exist constants $C_{j}$ and $C_{j}^{\prime}$ such that

$$
\begin{aligned}
& \sup _{y \in B_{R / 2}\left(x_{0}\right)}\left|\nabla^{j} u(y)\right| \\
& \quad \leq \frac{C_{j}\left\{1+R \rho\left(x_{0}, V\right)\right\}^{C_{j}^{\prime}}}{R^{j}} \sup _{y \in B_{R}\left(x_{0}\right)}|u(y)| .
\end{aligned}
$$

Proof. We use (58). From Lemma 12 (1) we have

$$
\begin{aligned}
\left|\nabla^{j} u\left(x_{0}\right)\right| \leq & C\left\{1+R \rho\left(x_{0}, V\right)\right\}^{2^{m+1} k_{0}} \\
& \times \rho\left(x_{0}, V\right)^{2^{m+1}} R^{2^{m+1}-j} \sup _{y \in B_{R}\left(x_{0}\right)}|u(y)| \\
& +\frac{C}{R^{j}}\left(\frac{1}{R^{n}} \int_{B_{R}\left(x_{0}\right)}|u(x)|^{2} d x\right)^{1 / 2} \\
\leq & \frac{C\left\{1+R \rho\left(x_{0}, V\right)\right\}^{2^{m+1}\left(k_{0}+1\right)}}{R^{j}} \sup _{y \in B_{R}\left(x_{0}\right)}|u(y)| .
\end{aligned}
$$

Then by the same argument as in the proof of Lemma 25, we arrive at the desired estimate.

The following theorem immediately follows from Lemmas 22 and 26.

Theorem 27. Let $m$ and $n$ be integers, $m \geq 0, n \geq 2^{m+1}+1$, and $1 \leq j \leq 2^{m+1}+1$. Suppose $V \in(R H)_{n / 2}$ and there exists $a$ positive constant $C$ such that $V(x) \leq C \rho(x, V)^{2}$. Then for any positive integer $N$ there exists a constant $C_{N}$ such that

$$
\left|\nabla_{x}^{j} \Gamma_{H_{2^{m}}}(x, y)\right| \leq \frac{C_{N}}{\{1+\rho(x, V)|x-y|\}^{N}} \cdot \frac{1}{|x-y|^{n-2^{m+1}+j}} .
$$

Using Theorem 27 we can prove the following theorem by the same way as the proof of Lemma 19.

Theorem 28. Let $j, m$, and $n$ be integers, $m \geq 0, n \geq 2^{m+1}+1$, and $0 \leq j \leq 2^{m+1}-1$. Suppose that $V \in(R H)_{n / 2}$ and there exists a positive constant $C$ such that $V(x) \leq C \rho(x, V)^{2}$. Then there exist positive constants $C_{j}$ such that (6) holds, where $1 \leq$ $p \leq \infty$.

We obtain the following pointwise estimate stated with the Hardy-Littlewood maximal operator.

Theorem 29. Let $j, m$, and $n$ be integers, $m \geq 0, n \geq 2^{m+1}+1$, and $0 \leq j \leq 2^{m+1}-1$. Suppose that $V \in(R H)_{n / 2}$ and there exists a positive constant $C$ such that $V(x) \leq C \rho(x, V)^{2}$. Then there exists a constant $C^{\prime}$ such that

$$
\left|V^{2^{m}-j / 2} \nabla^{j} H_{2^{m}}^{-1} f(x)\right| \leq C^{\prime} M f(x) .
$$

In Theorem 29, the cases $m=0$ and $m=1$ were shown in [5, Theorem 1.3] and [8, Lemma 10], respectively. 
Proof of Theorem 29. Let $r=1 / \rho(x, V)$. Since $V(x) \leq$ $C \rho(x, V)^{2}$, it follows from Theorems 9 and 27 that

$$
\begin{aligned}
& \left|V(x)^{2^{m}-j / 2} \nabla^{j} H_{2^{m}}^{-1} f(x)\right| \\
& \leq C C_{N} \int_{\mathbf{R}^{n}} \frac{\rho(x, V)^{2^{m+1}-j}|f(y)|}{\{1+\rho(x, V)|x-y|\}^{N}|x-y|^{n-2^{m+1}+j}} d y \\
& \leq C C_{N} \sum_{i=-\infty}^{\infty} \int_{2^{i-1} r<|x-y| \leq 2^{i} r}|f(y)| d y \\
& \leq C C_{N} \sum_{i=-\infty}^{\infty} \frac{\left(2^{i-1}\right)^{2^{m+1}-j}}{\left(1+2^{i-1}\right)^{N}} \\
& \quad \cdot \frac{1}{r^{2^{m+1}-j}\left(1+r^{-1}|x-y|\right)^{N}|x-y|^{n-2^{m+1}+j}} \int_{|x-y| \leq 2^{i} r}|f(y)| d y \\
& \leq C C_{N} \sum_{i=-\infty}^{\infty} \frac{\left(2^{i}\right)^{2^{m+1}-j}}{\left(1+2^{i}\right)^{N}} M f(x) .
\end{aligned}
$$

Then choosing $N \geq 2^{m+1}-j+1$ we arrive at the desired estimate.

Remark 30 (see [5, Remark 1.6]). Once we obtain the estimate (78), we can obtain boundedness of the operator $V^{2^{m}-j / 2} \nabla^{j} H_{2^{m}}^{-1}$ on any space on which $M$ is bounded, for example, Morrey spaces (see [12]).

\section{Conflict of Interests}

The author declares that there is no conflict of interests regarding the publication of this paper.

\section{Acknowledgments}

The author is grateful to the referee(s) for the helpful comment. The author is also grateful to Professor Kazuhiro Kurata, who read the first version of this paper and gave useful comment. This work was supported by Grant-in-Aid for Scientific Research (C) (no. 25400152), Japan Society for the Promotion of Science.

\section{References}

[1] J. Zhong, Harmonic analysis for some Schrödinger type operators [Ph.D. thesis], Princeton University, 1993.

[2] Y. Liu and J.-Z. Huang, " $L^{p}$ estimates for the Schrödinger type operators," Applied Mathematics-A Journal of Chinese Universities, vol. 26, no. 4, pp. 412-424, 2011.

[3] Z. W. Shen, " $L^{p}$ estimates for Schrödinger operators with certain potentials," Annales de l'Institut Fourier, vol. 45, no. 2, pp. 513546, 1995.
[4] J. Dziubański and J. Zienkiewicz, "Hardy space $H^{1}$ associated to Schrödinger operator with potential satisfying reverse Hölder inequality," Revista Matemática Iberoamericana, vol. 15, no. 2, pp. 279-296, 1999.

[5] K. Kurata and S. Sugano, "A remark on estimates for uniformly elliptic operators on weighted $L^{p}$ spaces and Morrey spaces," Mathematische Nachrichten, vol. 209, pp. 137-150, 2000.

[6] K. Kurata and S. Sugano, "Estimates of the fundamental solution for magnetic Schrödinger operators and their applications," The Tohoku Mathematical Journal, vol. 52, no. 3, pp. 367-382, 2000.

[7] S. Sugano, "Estimates for the operators $V^{\alpha}(-\Delta+V)^{-\beta}$ and $V^{\alpha} \nabla(-\Delta+V)^{-\beta}$ with certain nonnegative potentials $V$," Tokyo Journal of Mathematics, vol. 21, no. 2, pp. 441-452, 1998.

[8] S. Sugano, " $L^{p}$ estimates for some Schrödinger type operators and a Calderón-Zygmund operator of Schrödinger type," Tokyo Journal of Mathematics, vol. 30, no. 1, pp. 179-197, 2007.

[9] Y. Liu and J. Dong, "Some estimates of higher order Riesz transform related to Schrödinger type operators," Potential Analysis, vol. 32, no. 1, pp. 41-55, 2010.

[10] C. L. Fefferman, "The uncertainty principle," Bulletin of the American Mathematical Society, vol. 9, no. 2, pp. 129-206, 1983.

[11] F. W. Gehring, "The $L^{p}$-integrability of the partial derivatives of a quasiconformal mapping," Acta Mathematica, vol. 130, pp. 265-277, 1973.

[12] F. Chiarenza and M. Frasca, "Morrey spaces and HardyLittlewood maximal function," Rendiconti di Matematica e delle sue Applicazioni, vol. 7, no. 3-4, pp. 273-279, 1987. 


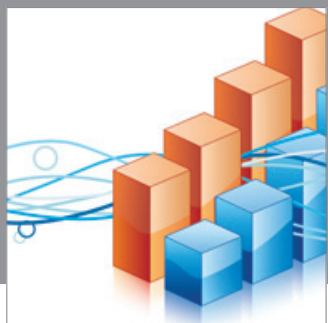

Advances in

Operations Research

mansans

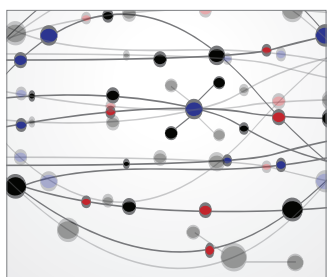

The Scientific World Journal
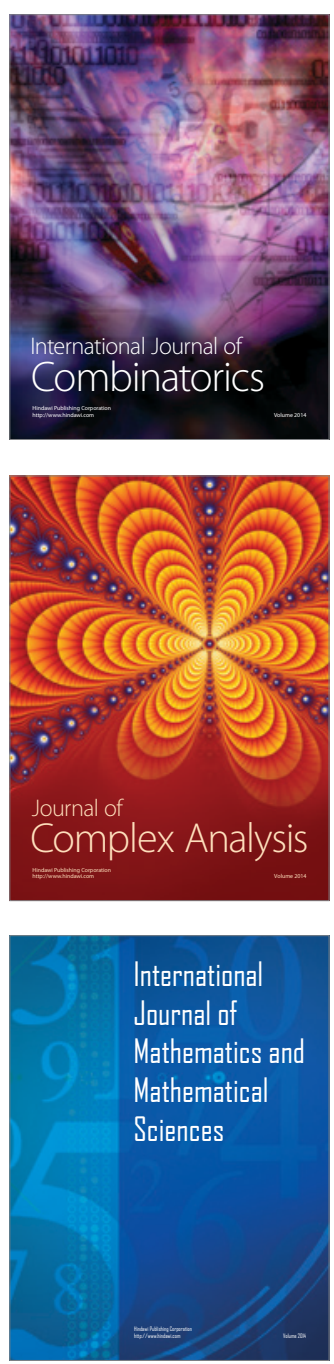
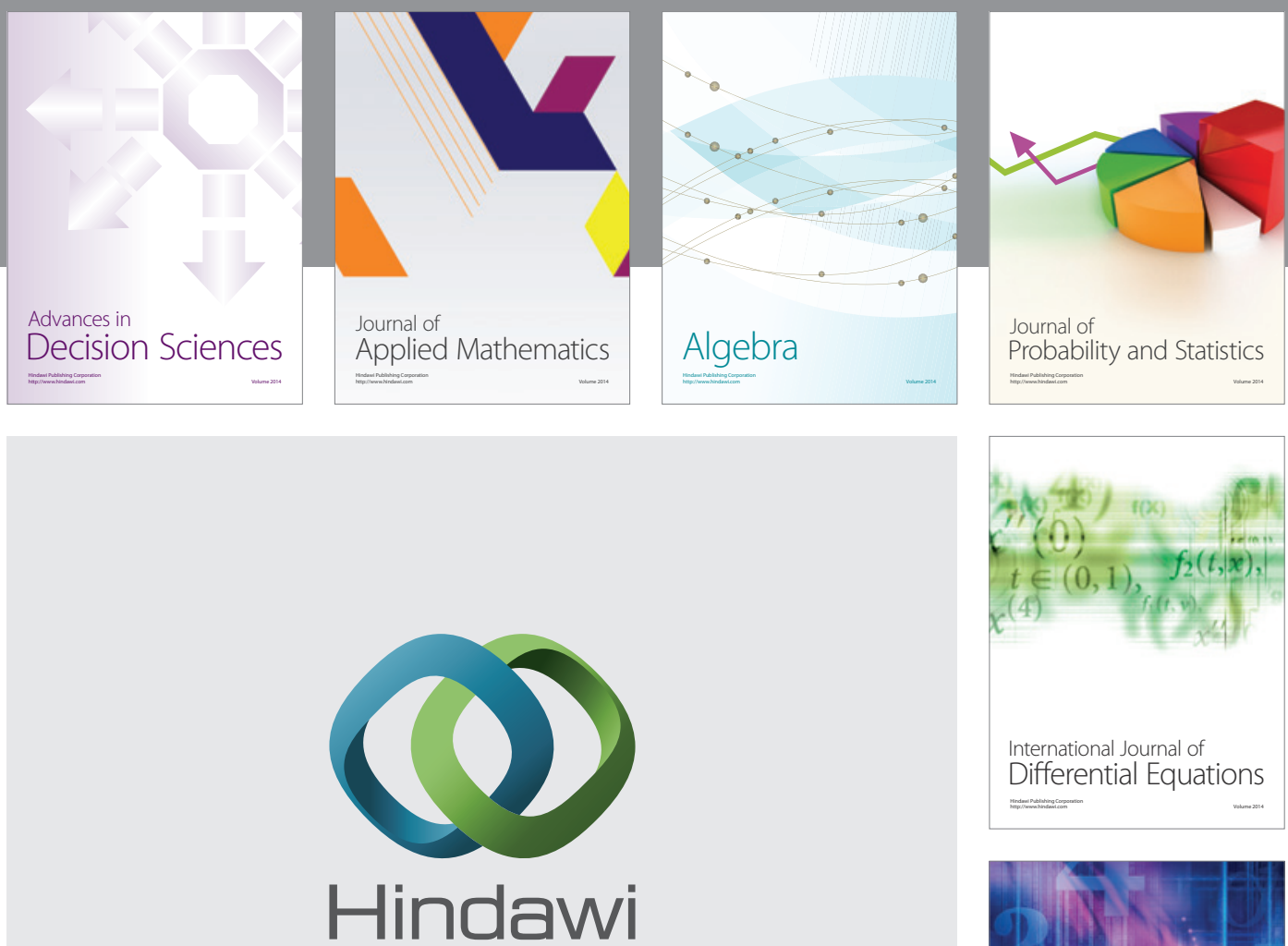

Submit your manuscripts at http://www.hindawi.com
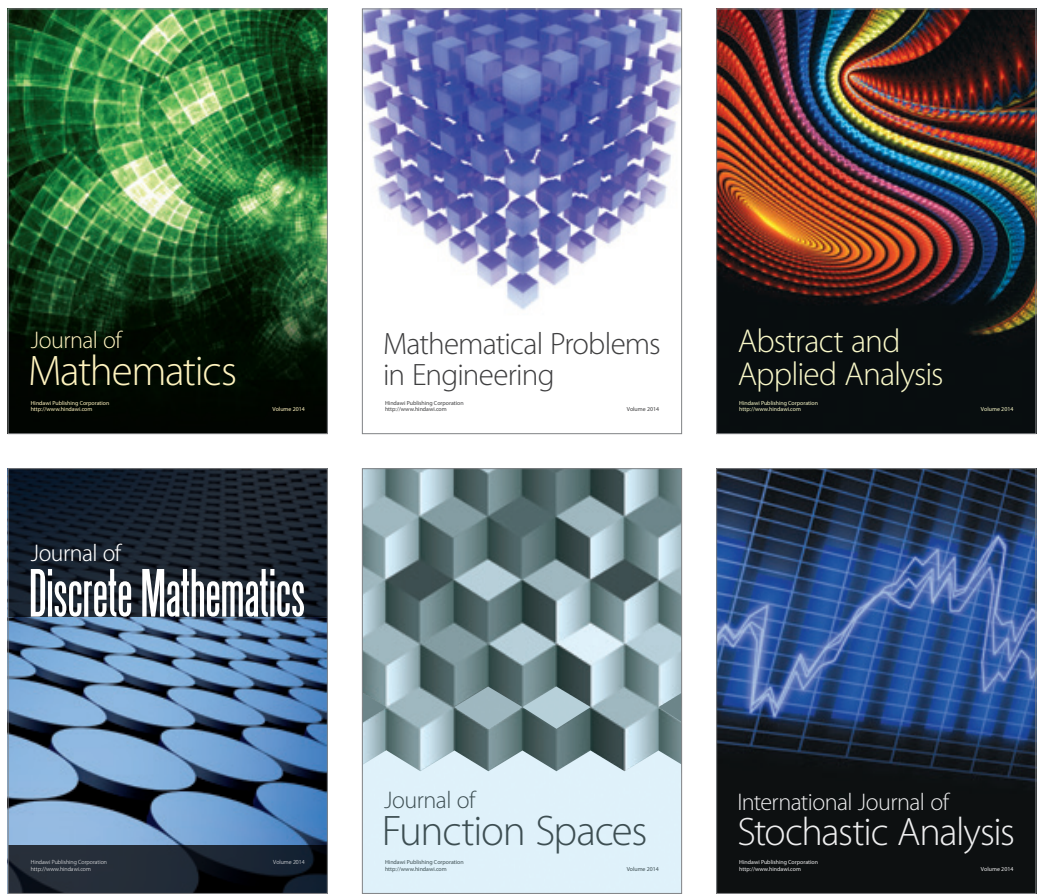

Journal of

Function Spaces

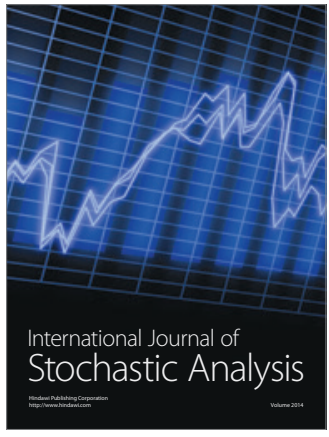

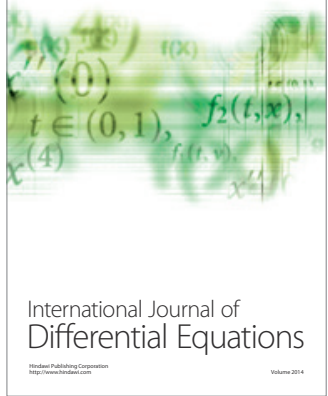
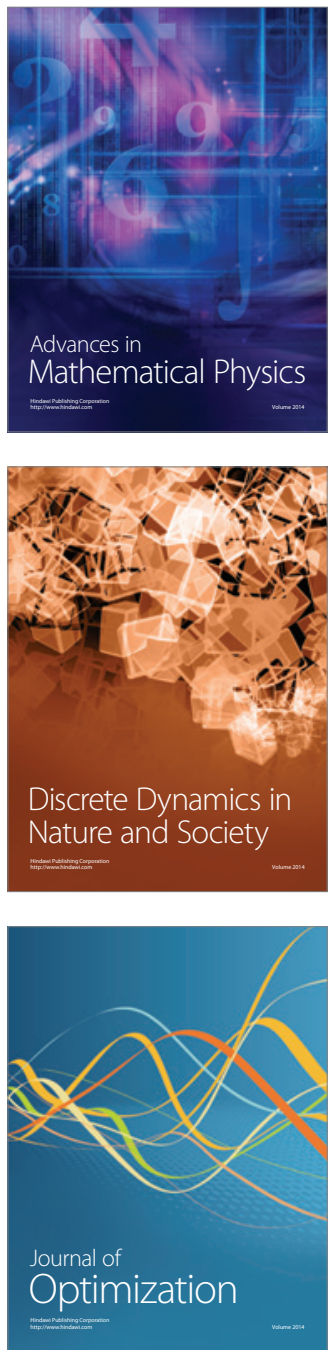